\title{
Fibrinogen does not relate to cardiovascular or muscle manifestations in COPD: cross-sectional data from the ERICA study
}

\author{
Divya Mohan, ${ }^{1,2}$ Julia R Forman, ${ }^{3}$ Matthew Allinder, ${ }^{2}$ Carmel M McEniery, ${ }^{3}$ \\ Charlotte Emma Bolton, ${ }^{4}$ John R Cockcroft, ${ }^{5}$ William MacNee, ${ }^{6}$ Jonathan Fuld, ${ }^{3}$ \\ Mellone Marchong, ${ }^{3}$ Nichola Sian Gale, ${ }^{5}$ Marie Fisk, ${ }^{3}$ Sridevi Nagarajan, ${ }^{3}$ \\ Joseph Cheriyan, ${ }^{3}$ David A Lomas, ${ }^{7}$ Peter M A Calverley, ${ }^{8}$ Bruce E Miller, ${ }^{2}$ \\ Ruth Tal-Singer, ${ }^{2}$ Ian B Wilkinson, ${ }^{3}$ Michael I Polkey, ${ }^{1}$ on behalf of the ERICA \\ Consortium
}

${ }^{1}$ NIHR Respiratory Biomedical Research Unit, Royal Brompton and Harefield NHS Foundation Trust, Imperial College, London, UK

${ }^{2} R \& D$, GlaxoSmithKline King of Prussia, Philadelphia, Pennsylvania, USA

${ }^{3}$ Division of Experimental Medicine and Immunotherapeutics, Cambridge Clinical Trials Unit, Addenbrooke's Hospital, University of Cambridge, Cambridge, UK

${ }^{4}$ Nottingham Respiratory Research Unit, NIHR Nottingham BRC, School of Medicine, City Hospital NUH Trust Campus, University of Nottingham, Nottingham, UK ${ }^{5}$ Department of Cardiology,

Wales Heart Research Institute, Cardiff University, Cardiff, UK

${ }^{6}$ MRC Centre for Inflammation, University of Edinburgh,

Edinburgh, UK

'Division of Medicine, UCL Respiratory, University College London, London, UK

${ }^{8}$ School of Ageing and Chronic Disease, University of Liverpool, Liverpool, UK

\section{Correspondence to} Dr Divya Mohan, R and D, GlaxoSmithKline King of Prussia, Philadelphia, PA 19406, USA; divya.x.mohan@gsk.com

RT-S, IBW and MIP contributed equally.

Received 18 January 2018 Revised 8 March 2018 Accepted 12 March 2018

Published Online First 4 April 2018

\section{Check for updates}

To cite: Mohan D,

Forman JR, Allinder $\mathrm{M}$, et al.

Thorax 2018;73:1182-1185.

\section{ABSTRACT}

Cardiovascular and skeletal muscle manifestations constitute important comorbidities in COPD, with

systemic inflammation proposed as a common mechanistic link. Fibrinogen has prognostic role in COPD. We aimed to determine whether aortic stiffness and quadriceps weakness are linked in COPD, and whether they are associated with the systemic inflammatory mediator-fibrinogen. Aortic pulse wave velocity (aPWV), quadriceps maximal volitional contraction (QMVC) force and fibrinogen were measured in 729 patients with stable, Global Initiative for Chronic Obstructive Lung Disease (GOLD) stages II-IV COPD. The cardiovascular and muscular manifestations exist independently $\left(P=0.22, \chi^{2}\right)$. Fibrinogen was not associated with aPWV or QMVC ( $P=0.628$ and $P=0.621$, respectively), making inflammation, as measured by plasma fibrinogen, an unlikely common aetiological factor.

\section{INTRODUCTION}

Cardiovascular disease and locomotor muscle dysfunction are two important comorbidities in COPD, occurring in up to $50 \%{ }^{12}$ of patients, and relating to both increased healthcare utilisation and mortality, thus representing potential therapeutic targets. Both share common risk factors, including smoking, exacerbations and physical inactivity, but systemic inflammation has been also proposed as a mechanistic link between them. If so, novel anti-inflammatory therapies might simultaneously target both manifestations. Fibrinogen is the only inflammatory biomarker with regulatory approval as a drug development tool in COPD, but hitherto the value of fibrinogen in predicting these phenotypes has not been examined.

In the 'Evaluating the Role of Inflammation in Chronic Airways disease' (ERICA) study, we investigated whether cardiovascular and skeletal muscle manifestations overlap in COPD and whether these manifestations are associated with plasma fibrinogen. ERICA is unique among multicentre studies in objectively quantifying these non-pulmonary manifestations of COPD.

\section{METHODS}

Methods have been described previously. ${ }^{3}$ ERICA is a prospective, observational study that recruited subjects from hospital and community settings, and was registered with the UK Clinical Research Network Study Portfolio (http://public.ukcrn.org.uk/ Search/StudyDetail.aspx?StudyID=11101). Participants provided written consent to participate.

Subjects were aged 40-90 years with Global Initiative for Chronic Obstructive Lung Disease (GOLD) stages II-IV COPD judged by postbronchodilator spirometry. Relevant exclusion criteria were alpha-1-antitrypsin deficiency or significant coexisting pulmonary disease; subjects were clinically stable for $>4$ weeks. Comorbid disease was not an exclusion criterion.

Subjects underwent multiple phenotypic assessments, including aortic pulse wave velocity (aPWV), quadriceps maximal volitional contraction (QMVC) force, 6 min walk distance (6MWD) and fibrinogen.

APWV of $>10 \mathrm{~m} / \mathrm{s}$ was chosen as a surrogate measure of cardiovascular disease. ${ }^{4}$ Quadriceps weakness was defined according to the gender-specific Seymour equation. ${ }^{2}$ The 'Cardiovascular' group contained subjects with elevated aPWV alone, 'Muscle' contained subjects with skeletal muscle weakness only, 'Both' contained subjects with both manifestations and 'Neither' had subjects with neither manifestation.

Multiple linear regression analyses were conducted to estimate the dependence of QMVC and aPWV on fibrinogen, with adjustment for known or expected confounders. Non-normally distributed variables were $\log$-transformed prior to analysis.

$\mathrm{X}^{2}$ testing was carried out to test whether QMVC and aPWV were independently associated.

Analyses were performed using R V.3.0.0 for Microsoft Windows with RStudio V.0.98.953.

\section{RESULTS}

Seven hundred and forty-six people were screened; 729 met the inclusion criteria and 617 subjects had both aPWV and QMVC measurements. Table 1 describes the baseline demographics for the whole cohort and phenotypic groups. The population had a mean age of 67 years, $61 \%$ were male, with a mean $\mathrm{FEV}_{1}$ of $52.2 \%$ predicted, mean aPWV of $10.3 \mathrm{~m} / \mathrm{s}$ and mean QMVC of $30.6 \mathrm{~kg}$ (68.2\% predicted).

Of 617 subjects, 194 (31.4\%) were classified 


\begin{tabular}{|c|c|c|c|c|c|}
\hline Variable & Overall $(n=729)$ & Neither $(n=194)$ & Muscle $(n=137)$ & Cardiovascular $(n=154)$ & Both $(n=132)$ \\
\hline Age (years) & $67(8)$ & $66(7)^{* * *}$ & $63(8)^{* * *}$ & $70(7)^{* * *}$ & $70(7)^{* * *}$ \\
\hline Gender (\% male) & 60.6 & 58.3 & 54.7 & 67.5 & 62.9 \\
\hline Smoking history (total pack years) & $47(28)$ & $45(27)$ & $47(26)$ & $51(31)$ & $47(28)$ \\
\hline BMI $\left(\mathrm{kg} / \mathrm{m}^{2}\right)$ & $27.3(5.8)$ & $26.3(4.9)^{* *}$ & $26.9(5.6)$ & $27.1(4.9)$ & $28.3(6.5)^{*}$ \\
\hline Fat-free mass $(\mathrm{kg})$ & $51.0(11.1)$ & $49.7(10.6)$ & $50.1(11.2)$ & $51.9(10.5)$ & $52.2(11.9)$ \\
\hline CAT score $(0-40)$ & $20(0-40)$ & $17(0-38)^{* \star *}$ & $22(1-39)^{* *}$ & $19(3-40)$ & $21.5(6-40)^{*}$ \\
\hline SGRQ total score & $51.4(22.3)$ & $44.2(21.2)^{\star * *}$ & $57.4(21.5)^{* \star *}$ & $47.7(22.5)^{*}$ & $55.8(20.5)^{*}$ \\
\hline $\mathrm{FEV}_{1}(\mathrm{~L})$ & $1.34(0.53)$ & $1.50(0.52)^{\star * *}$ & $1.31(0.53)$ & $1.32(0.50)$ & $1.26(0.50)$ \\
\hline $\mathrm{FEV}_{1}(\%$ predicted $)$ & $52.2(16.1)$ & $57.4(14.2)^{* * *}$ & $49.4(16.3)^{*}$ & $51.8(16.4)$ & $50.5(15.8)$ \\
\hline $\mathrm{FEV}_{1} / \mathrm{FVC}$ & $0.48(0.13)$ & $0.49(0.12)$ & $0.48(0.13)$ & $0.47(0.12)$ & $0.49(0.13)$ \\
\hline \multicolumn{6}{|l|}{ Muscle indices } \\
\hline QMVC (kg) & $30.6(11.5)$ & $36.1(10.1)$ & $23.4(7.9)$ & $36.7(10.7)$ & $22.7(8.2)$ \\
\hline QMVC (\% predicted) & $68.2(20.7)$ & $81.4(15.3)$ & $51.5(12.0)$ & $82.2(14.9)$ & $50.2(12.9)$ \\
\hline SPPB total score & $10(1-12)$ & $11(3-12)^{* \star *}$ & $10(3-12)$ & $11(4-12)^{* *}$ & $9(2-12)^{* * *}$ \\
\hline 6MWD (m) & $345(130)$ & $404(104)^{* \star \star}$ & $338(129)$ & $361(125)$ & $282(132)^{* * *}$ \\
\hline \multicolumn{6}{|l|}{ Cardiovascular indices } \\
\hline Supine MAP (mm Hg) & $98(12)$ & $96(11)^{*}$ & $95(10)^{* \star *}$ & $100(10)^{*}$ & $100(11)^{*}$ \\
\hline $\mathrm{aPWV}(\mathrm{m} / \mathrm{s})$ & $10.3(2.6)$ & $8.3(1.1)$ & $8.3(1.1)$ & $12.2(2.0)$ & $12.7(1.9)$ \\
\hline \multicolumn{6}{|l|}{ Inflammatory markers } \\
\hline Fibrinogen $(\mathrm{g} / \mathrm{dL})$ & $3.49(0.86)$ & $3.44(0.95)$ & $3.41(0.76)$ & $3.49(0.78)$ & $3.52(0.90)$ \\
\hline hsCRP (mg/L) & $6.57(12.1)$ & $7.45(19.7)$ & $6.11(8.13)$ & $5.63(6.95)$ & $6.365(6.55)$ \\
\hline WCC $\left(\times 10^{9} / \mathrm{L}\right)$ & $7.50(2.16)$ & $7.32(2.15)$ & $7.19(2.18)$ & $7.42(1.95)$ & $7.70(2.38)$ \\
\hline \multicolumn{6}{|l|}{ Comorbidities } \\
\hline Exacerbations per year & $1.9(2.1)$ & $1.71(1.8)$ & $2.2(2.0)^{*}$ & $1.81(2.34)$ & $2.2(2.3)^{*}$ \\
\hline Diabetes (\%) & 11.4 & 6.3 & 7.2 & 10.4 & $18.3^{* *}$ \\
\hline Antihypertensive therapy (\%) & 35.9 & 31.4 & 28.9 & 39.0 & $43.9^{*}$ \\
\hline Cholesterol-lowering therapy (\%) & 32.6 & 22.8 & 28.5 & $37.9^{* *}$ & $43.8^{* * *}$ \\
\hline Previous myocardial infarction (\%) & 7.9 & 6.3 & 6.6 & 9.1 & 9.2 \\
\hline
\end{tabular}

Baseline demographics for overall cohort ( $\mathrm{n}=729$ ) and phenotypic groups. Values expressed as mean (SD) for continuous variables and median (range) for discrete variables. The 'Neither' group ( $n=194)$ represents subjects with neither elevated aPWV nor skeletal muscle weakness. The 'Muscle' group ( $n=137)$ represents subjects with skeletal muscle weakness alone. The 'Cardiovascular' group ( $n=154$ ) represents subjects with cardiovascular manifestation, defined as elevated aPWV, alone, and the 'Both' group ( $n=132$ ) contains subjects demonstrating both skeletal muscle weakness and elevated aPWV. Skeletal muscle weakness according to predicted QMVC based on equations developed by Seymour et $a l^{2}$ and elevated aPWV defined as $>10 \mathrm{~m} / \mathrm{s}$.

${ }^{*} \mathrm{P}<0.05,{ }^{* *} \mathrm{P}<0.01,{ }^{* *} \mathrm{P}<0.001$ for significant differences across groups. For continuous variables, the $\mathrm{P}$ value represents differences between overall mean and group mean. For ordinal and binary variables, the P value represents differences between the 'Neither' group and the remaining groups. Adjustments have not been made for multiple comparisons.

6MWD, 6 min walk distance; aPWV, aortic pulse wave velocity; BMI, body mass index; CAT, COPD Assessment Test; hsCRP, high-sensitivity C reactive protein; MAP, mean arterial pressure; QMVC, quadriceps maximal volitional contraction; SGRQ, St George's Respiratory Questionnaire; SPPB, short physical performance battery; WCC, white cell count.

as 'Neither', 137 (22.2\%) as 'Muscle', $154(30.0 \%)$ as 'Cardiovascular' and $132(21.4 \%)$ as 'Both' (supplementary eFigure 1). Overall, 269 out of $617(43.6 \%)$ subjects had muscle weakness and 286 out of $617(46.4 \%)$ had elevated aPWV. $X^{2}$ test confirmed that cardiovascular and skeletal muscle manifestations were independent (test statistic 1.49; $\mathrm{P}=0.22$ ).

Fibrinogen was not siginificantly associated with either APWV or QMVC ( $\mathrm{P}=0.628$ and $\mathrm{P}=0.621$ ) (figure 1, supplementary eTable 2 and eTable 3 ).

Age, gender, supine heart rate, supine mean arterial pressure and diabetes were significantly associated with aPWV (online supplementary eTable 2). Smoking history, $\mathrm{FEV}_{1}$, previous self-reported myocardial infarction, antihypertensive therapy and cholesterol-lowering therapy were not significantly associated.

QMVC was significantly and independently associated with age, gender, fat-free mass and $\mathrm{FEV}_{1}$ (online supplementary eTable 3). Smoking history and height were not significant associations.

\section{DISCUSSION}

Cardiovascular and skeletal muscle manifestations are more prevalent in COPD than previously reported, but do not coexist more frequently than is expected by chance and therefore likely require different therapeutic approaches. Furthermore, fibrinogen was not associated with quadriceps strength or aortic stiffness, suggesting that it is unlikely to be useful in clinical trials addressing cardiovascular or skeletal muscle manifestations in COPD. Our findings do not support the concept that anti-inflammatory therapy aiming to reduce fibrinogen is 


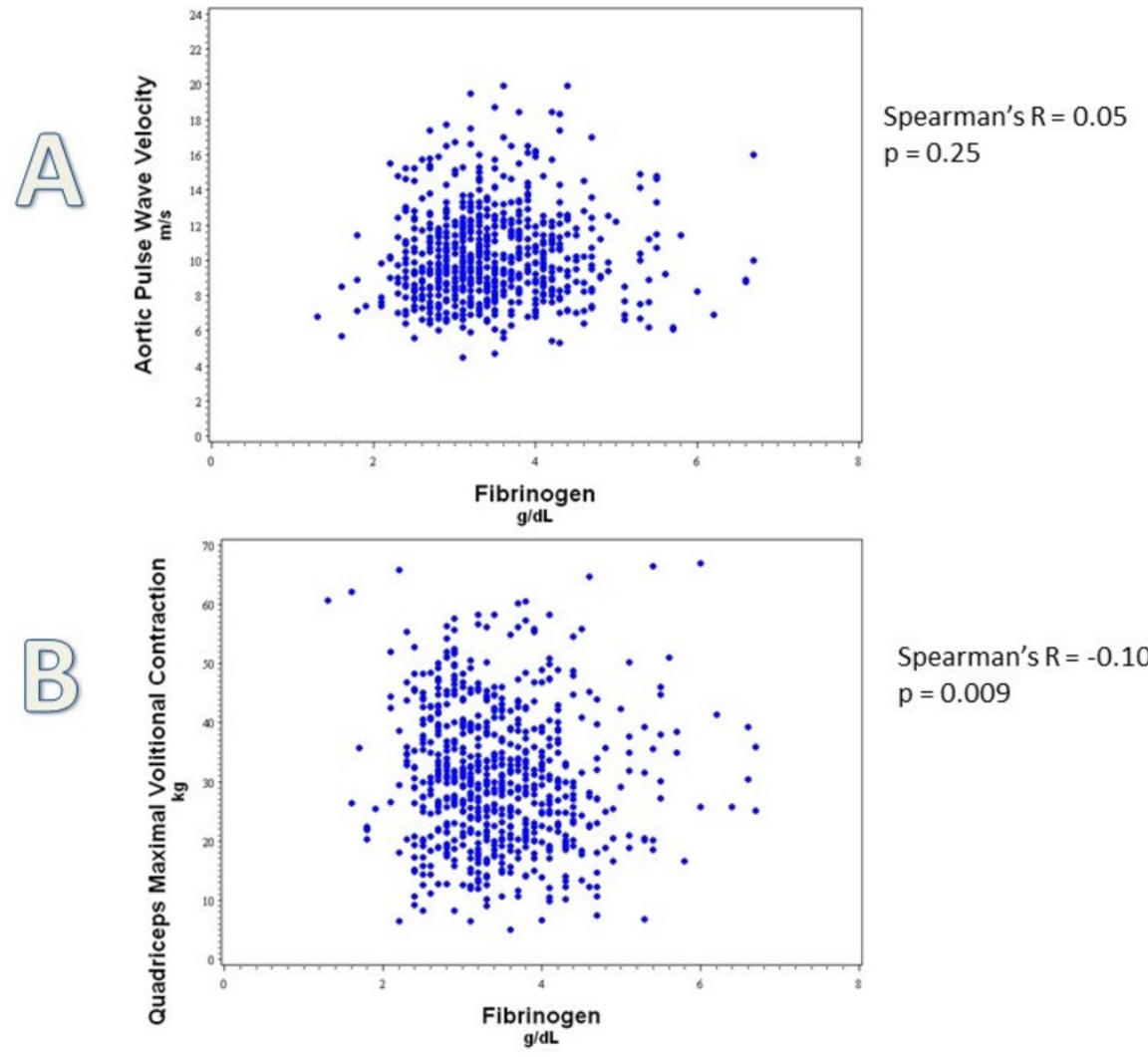

Figure 1 Relationship between fibrinogen and aPWV and QMVC. Scatterplot demonstrating relationship between (A) fibrinogen ( $\mathrm{g} / \mathrm{L}$ ) and arterial stiffness, as measured by aPWV (m/s) in 651 subjects with COPD, with Spearman's correlation of 0.05 and $\mathrm{P}=0.25$; and $(\mathrm{B})$ fibrinogen ( $\mathrm{g} / \mathrm{L})$ and quadriceps weakness, as measured by QMVC $(\mathrm{kg})$ in 684 subjects with COPD, with Spearman's correlation -0.10 and $\mathrm{P}=0.009$. aPWV, aortic pulse wave velocity; QMVC, quadriceps maximal volitional contraction.

likely to reduce aortic stiffness or improve quadriceps strength in COPD.

Our estimate of cardiovascular comorbidity agrees with previous estimates. Associations with aPWV in our patients were similar to those previously observed in healthy subjects. ${ }^{4}$ Interestingly fibrinogen was not associated with arterial stiffness, despite prior reports of association of aPWV with interleukin-6 and C reactive protein (CRP); moreover, we failed to find associations between white cell count, CRP and aPWV or QMVC (online supplementary eTables 4-13). Furthermore, we did not find an association between FEV and aPWV, in contrast to smaller studies, ${ }^{5}$ suggesting that aPWV is not linked to the severity of airflow limitation in people diagnosed with COPD.

In our cohort, the prevalence of quadriceps weakness (43.6\%) is higher than previously reported. ${ }^{2}$ In COPD, muscle weakness precedes the development of whole body cachexia, with relative upper limb sparing ${ }^{6}$; therefore, measures other than QMVC (eg, hand grip strength or bioimpedance) are likely to underestimate the prevalence of muscle dysfunction.

This study is one of the few to have examined the coexistence of cardiac and muscle manifestations. Using different methodologies prior studies found lower prevalences; Vanfleteren et $a l^{7}$ reported $10 \%$ of patients in their cardiovascular cluster had muscle wasting while $7 \%$ of patients with cachexia had a history of myocardial infarction. Siebeling et $\mathrm{l}^{8}$ estimate the coexistence of cardiovascular and muscular comorbidities at 2\%-3\%. Of our cohort, $21.4 \%$ exhibited both elevated aPWV and quadriceps weakness; $\chi^{2}$ testing confirmed that there is not an independent, overlap syndrome.
Our study supports recent data that refute the link between low-grade systemic inflammation and cardiovascular or muscle manifestations in COPD. ${ }^{79}$ Persistent systemic inflammation is present in $16 \%$ of patients with $\mathrm{COPD}^{10}$; given that we report prevalence of both aPWV and QMVC is much higher than 16\%, inflammation seems an improbable key aetiological factor. The cross-sectional nature of our data means that a temporal link between PWV and QMVC or systemic inflammation cannot be assessed at this stage, although follow-up of the cohort is ongoing. Additionally, it is important to note that our study allowed the cross-sectional assessments to be carried out over either a single visit or two visits within a 3-month period; therefore, we acknowledge that in some patients inflammatory markers were measured on a separate day to aPWV and QMVC. A further limitation is that 112 patients of the entire data set did not have the three key measures assessed here. These patients were similar in most respects to the cohort analysed here but had a lower $\mathrm{FEV}_{1}$ and $6 \mathrm{MWD}$ (data not shown).

In conclusion, cardiovascular and skeletal muscle manifestations are prevalent in COPD, with muscle weakness being more common than previously thought. These extrapulmonary manifestations are distinct and need to be addressed individually. Neither aortic stiffness nor quadriceps weakness relates to plasma fibrinogen, suggesting that this is not a useful biomarker for trials addressing these manifestations and that inflammation is an unlikely aetiological factor.

Acknowledgements The authors would like to thank the subjects who participated in the study, as well as the study teams at all five participating centres, especially Jessica Middlemiss (Cambridge Clinical Trials Unit) and Cayley Smith 
(Muscle Lab, Royal Brompton Hospital), who were instrumental in helping to set up the study.

Contributors MIP, DM, JRF, CMM, CEB, JRC, WM, JF, NSG, MF, JRC, DAL, PMAC, $B E M, R T-S$ and IBW all made substantial contributions to the conception and design of the work. MIP, DM, JRF, MA, CMM, CEB, JRC, WM, JF, MM, SN, NSG, MF and IBW made substantial contributions to the acquisition and analysis of data for the work. All authors contributed to the interpretation of the data, drafting and revision of the manuscript, and final approval of the version submitted for publication. All authors take accountability for all aspects of the work.

Funding This study was funded by a grant from Innovate UK. GlaxoSmithKline, a consortium partner, made contributions in kind towards study management. The UK Clinical Research Network contributed towards the study at all participating sites. Subjects from the Wales Heart Research Institute, Cardiff, were simultaneously recruited to the ARCADE study (ClinicalTrials.gov Identifier NCT01656421), which was funded by GSK. IBW, JRC and CMM have received funding support from the National Institute for Health Research (NIHR) Cambridge Comprehensive Biomedical Research Centre. Part of the work was undertaken at the NIHR Respiratory Biomedical Research Unit at the Royal Brompton and Harefield NHS Foundation Trust and Imperial College London, which part-funded DM and MIP's salary.

Competing interests MIP has received payment to his institution or himself for advice on skeletal muscle weakness in COPD from GSK, Novartis, AZ, Pfizer, Lilly and Astellas. DM is an employee and shareholder of GSK. JRF, CMM, MM, SN and NSG have no conflict of interest to report. CEB reports grants from Innovate UK (formerly Technology Strategy Board (TSB) UK) during the conduct of the study; advisory board fees from GSK paid to their institution, grants from GSK and grants from MRC/ABPI outside the submitted work. JRC reports grants from TSB/MRC, during the conduct of the study; personal fees from GSK, outside the submitted work. WM received research support from GlaxoSmithKline and Pfizer, and was on advisory committees of Almirall, GlaxoSmithKline, Novartis and Pfizer; he was a speaker for AstraZeneca, Boehringer Ingelheim, GlaxoSmithKline, Janssen and Novartis. Jf received speaker fees from GSK. MF acknowledges receipt of an imaging fellowship award from GSK. JRC is employed full-time by Cambridge University Hospitals National Health Service Foundation Trust and is obligated to spend $50 \%$ of his time on GSK clinical trial research, representing a significant relationship; however, he receives no other benefits or compensations from GSK. DAL reports grants and personal fees from GSK, and personal fees from Grifols, outside the submitted work. PMAC has advised Boehringer Ingelheim, GSK, AstraZeneca and Takeda on the design and conduct of clinical trials, and has spoken at meetings sponsored by these companies and by
Novartis; he has no stock holdings in any pharmaceutical company or connection with the tobacco industry. BEM and RT-S are shareholders and employees of GSK. IBW reports grants from TSB and GSK during the conduct of the study, and grants from GSK outside the submitted work.

Patient consent Obtained.

Ethics approval The Cambridge South Research Ethics Committee (reference 11/ EE/0357) approved the study.

Provenance and peer review Not commissioned; internally peer reviewed.

(c) Article author(s) (or their employer(s) unless otherwise stated in the text of the article) 2018. All rights reserved. No commercial use is permitted unless otherwise expressly granted.

\section{REFERENCES}

1 Divo M, Cote C, de Torres JP, et al. Comorbidities and risk of mortality in patients with chronic obstructive pulmonary disease. Am J Respir Crit Care Med 2012;186:155-61.

2 Seymour JM, Spruit MA, Hopkinson NS, et al. The prevalence of quadriceps weakness in COPD and the relationship with disease severity. Eur Respir J 2010;36:81-8.

3 Mohan D, Gale NS, McEniery CM, et al. Evaluating the role of inflammation in chronic airways disease: the ERICA study. COPD 2014;11:552-9.

4 Reference Values for Arterial Stiffness' Collaboration. Determinants of pulse wave velocity in healthy people and in the presence of cardiovascular risk factors: 'establishing normal and reference values'. Eur Heart J 2010;31:2338-50.

5 Vivodtzev I, Tamisier R, Baguet JP, et al. Arterial stiffness in COPD. Chest 2014;145:861-75.

6 Bernard S, LeBlanc P, Whittom F, et al. Peripheral muscle weakness in patients with chronic obstructive pulmonary disease. Am J Respir Crit Care Med 1998;158:629-34.

7 Vanfleteren LE, Spruit MA, Groenen M, et al. Clusters of comorbidities based on validated objective measurements and systemic inflammation in patients with chronic obstructive pulmonary disease. Am J Respir Crit Care Med 2013:187:728-35.

8 Siebeling L, Puhan MA, Muggensturm P, et al. Characteristics of Dutch and Swiss primary care COPD patients - baseline data of the ICE COLD ERIC study. Clin Epidemiol 2011;3:273-83.

9 Vanfleteren LE, Spruit MA, Groenen MT, et al. Arterial stiffness in patients with COPD: the role of systemic inflammation and the effects of pulmonary rehabilitation. Eur Respir J 2014;43:1306-15.

10 Agustí A, Edwards LD, Rennard SI, et al. Persistent systemic inflammation is associated with poor clinical outcomes in COPD: a novel phenotype. PLoS One 2012;7:e37483. 